\title{
Caracterização de sinais e sintomas de câncer infantojuvenil: A percepção dos pais de acometidos
}

\author{
Characterization of signs and symptoms of childhood cancer: The perception of the parents of \\ affected children \\ Caracterización de los signos y síntomas del cáncer infantil y adolescente: La percepción de los \\ padres de los niños afectados
}

Recebido: 02/07/2021 | Revisado: 07/07/2021 | Aceito: 08/07/2021 | Publicado: 21/07/2021

\author{
Yasmin Oliveira Santos \\ ORCID: https://orcid.org/0000-0003-0677-1263 \\ Universidade Tiradentes, Brasil \\ E-mail: yasmin_os@hotmail.com \\ Manuela Naiane Lima Barreto \\ ORCID: https://orcid.org/0000-0002-4487-9227 \\ Universidade Tiradentes, Brasil \\ E-mail: manuela.naiane@gmail.com \\ Beatriz Aguiar da Mota \\ ORCID: https://orcid.org/0000-0001-8093-0205 \\ Universidade Tiradentes, Brasil \\ E-mail: beatriz.aguiarmt2018@gmail.com \\ Rute Nascimento da Silva \\ ORCID: https://orcid.org/0000-0002-2719-1623 \\ Universidade Tiradentes, Brasil \\ E-mail: silva_rute@hotmail.com \\ Felipe Mendes de Andrade de Carvalho \\ ORCID: https://orcid.org/0000-0002-7360-7925 \\ Universidade Tiradentes, Brasil \\ E-mail: felipemdadc@gmail.com \\ Carla Viviane Freitas de Jesus \\ ORCID: https://orcid.org/0000-0002-7775-6610 \\ Universidade Tiradentes, Brasil \\ E-mail: carlavfj@gmail.com \\ Ana Jovina Barreto Bispo \\ ORCID: https://orcid.org/0000-0002-6228-768X \\ Universidade Tiradentes, Brasil \\ E-mail: anajovina70@gmail.com
}

\begin{abstract}
Resumo
Objetivo: Conhecer os sinais e sintomas do câncer infantojuvenil percebidos pelos pais dos acometidos e o tempo entre procura de um serviço de saúde e o diagnóstico da doença. Metodologia: Estudo transversal, de caráter exploratório e abordagem quantitativa, através de banco de dados cadastrais, padronizado pela AVOSOS, realizado no período de dezembro de 2019, que incluem os genitores de crianças e adolescentes, entre 0 a 19 anos, em tratamento para neoplasias malignas, assistidos pela instituição, no momento da admissão. Resultados: Participaram do estudo 719 crianças e adolescentes. A febre $(40 \%)$ e a dor $(38,1 \%)$ foram os sinais e sintomas mais relatados pelos genitores, seguidos por cefaleia, vômitos, astenia e tumorações. A procura pelo atendimento médico ocorreu dentro de um mês em 79,6\% e diagnóstico do câncer ocorreu de 1 a 3 meses do início dos sintomas em 39,9\%. A leucemia e o linfoma foram as neoplasias mais frequentes. Neuroblastoma, tumores ósseos, tumores do SNC, tumores de tecidos moles e outros sarcomas foram os maiores responsáveis pelos óbitos. Os tratamentos mais realizados pelos portadores de CIJ foram, respectivamente, a quimioterapia e os procedimentos cirúrgicos. Conclusão: A febre, uma das manifestações iniciais da leucemia, a neoplasia mais comum, é o principal sintoma que leva ao atendimento médico. A procura por um serviço médico ocorreu em até um mês da ocorrência dos sintomas e diagnóstico entre um a três meses após o início da sintomatologia, no entanto, merece preocupação a procura após um mês do primeiro sintoma em uma parcela significativa da população.
\end{abstract}

Palavras-chave: Neoplasias; Sinais e sintomas; Criança; Adolescente; Epidemiologia.

\section{Abstract}

Objective: To know the signs and symptoms of childhood and adolescent cancer perceived by the parents of those affected and the time between seeking a health service and the diagnosis of the disease. Methodology: Cross-sectional 
study, of exploratory nature and quantitative approach, through registration database, standardized by AVOSOS, carried out in the period of December 2019, which includes the parents of children and adolescents, between 0 and 19 years old, in treatment for malignant neoplasms, assisted by the institution, at the time of admission. Results: 719 children and adolescents participated in the study. Fever $(40 \%)$ and pain $(38.1 \%)$ were the signs and symptoms most reported by parents, followed by headache, vomiting, asthenia and tumors. Seeking medical care occurred within one month in $79.6 \%$ and cancer diagnosis occurred within 1 to 3 months of symptom onset in $39.9 \%$. Leukemia and lymphoma were the most frequent neoplasms. Neuroblastoma, bone tumors, CNS tumors, soft tissue tumors, and other sarcomas were most responsible for deaths. The most common treatments performed for CIJ patients were chemotherapy and surgical procedures, respectively. Conclusion: Fever, one of the initial manifestations of leukemia, the most common neoplasm, is the main symptom leading to medical attention. Seeking medical service occurred within one month of symptom occurrence and diagnosis between one and three months after symptom onset, however, seeking after one month of first symptom in a significant portion of the population merits concern.

Keywords: Neoplasms; Signs and Symptoms; Child; Adolescent; Epidemiology.

\section{Resumen}

Objetivo: Conocer los síntomas del cáncer infantojuvenil percibidos por los acometidos y el tiempo que transcurre entre la solicitud de un servicio de salud y el diagnóstico de la enfermedad. Metodología: Estudio transversal, de carácter exploratorio y de abordaje cuantitativo, a través de banco de datos catastrales, patrocinado por la AVOSOS, realizado en el período de diciembre de 2019, que incluyó a los genitores de niños y adolescentes, entre 0 y 19 años, en tratamiento para neoplasias malignas, asistidos por la institución, en el momento del ingreso. Resultados: 719 niños y adolescentes participaron en el estudio. La fiebre $(40 \%)$ y el dolor $(38,1 \%)$ fueron los signos y síntomas más señalados por los padres, seguidos de la cefalea, los vómitos, la astenia y los tumores. La búsqueda de atención médica se produjo en el plazo de un mes en el 79,6\% y el diagnóstico de cáncer se produjo entre 1 y 3 meses después del inicio de los síntomas en el 39,9\%. La leucemia y el linfoma fueron las neoplasias más frecuentes. El neuroblastoma, los tumores óseos, los tumores del SNC, los tumores de tejidos blandos y otros sarcomas fueron los más responsables de las muertes. Los tratamientos más comunes realizados por los pacientes con CIJ fueron, respectivamente, la quimioterapia y los procedimientos quirúrgicos. Conclusión: La fiebre, una de las manifestaciones iniciales de la leucemia, la neoplasia más común, es el principal síntoma que conduce a la atención médica. La búsqueda de un servicio médico se produjo en el plazo de un mes desde la aparición de los síntomas y el diagnóstico entre uno y tres meses después de la aparición de los síntomas, sin embargo, merece preocupación la búsqueda después de un mes del primer síntoma en una parte significativa de la población.

Palabras clave: Neoplasias; Signos y Síntomas; Niño; Adolescente; Epidemiología.

\section{Introdução}

O câncer infantojuvenil é um importante problema de saúde pública pelo grande impacto físico e psicossocial nos pacientes e famílias afetadas (Ries \& Costenaro, 2017; Lilja-Fischer, Schrøderc \& Nielsend, 2019). Corresponde a 1 a 4\% de todos os tumores na maioria das populações, representando nos países em desenvolvimento o equivalente a 3 a $10 \%$ do total de cânceres. No Brasil, o percentual médio de neoplasias nos Registros de Câncer de Base Populacional (RCBP) na população infantojuvenil (de 0 a 19 anos) foi de 3\% (Brasil, 2017). As neoplasias mais frequentes nesta faixa etária são as leucemias, seguidas pelos tumores do sistema nervoso central (SNC) e linfomas (Feliciano, Santos \& Pombo-de-Oliveira, 2018).

A Classificação Internacional de Câncer Infantil (CICI) inclui 12 grupos tumorais principais e 47 subgrupos incluindo apenas tumores considerados malignos, com exceção do grupo III, onde também estão inseridos tumores intracranianos ou intra-espinhais de histologia benigna. Os 12 grupos principais são: I leucemias, II linfomas e neoplasmas reticulares, III tumores do sistema nervoso central, IV neuroblastoma e outros tumores de células nervosas periféricas, V retinoblastoma, VI tumores renais, VII tumores hepáticos, VIII tumores ósseos malignos, IX sarcomas de tecido mole, X Tumores germinativos, trofoblásticos e outros tumores gonadais, XI outra neoplasia epitelial e melanoma e XII outras neoplasias especificadas e neoplasia maligna não especificada (Bravo et al., 2013).

Apesar de não representar doença de elevada incidência, tem crescido cerca de $1 \%$ ao ano no mundo. Nesse contexto, estima-se que o CIJ afete aproximadamente 150-200 por 1 milhão de crianças por ano (Lilja-Fischer, Schrøderc \& Nielsend, 2019), sendo que no Brasil foram computados 12600 novos casos de câncer em crianças e adolescentes até os 19 anos no ano 
de 2016 (Ries \& Costenaro, 2017) e nos Estados Unidos, antes de atingirem a idade adulta, 1 em cada 600 crianças desenvolvem algum tipo de câncer (Radhi et al., 2015).

Além disso, o CIJ é a principal causa de óbito infantil em países desenvolvidos e os sobreviventes possuem uma elevada taxa de morbidade e risco de malignidade secundária a longo prazo (Lilja-Fischer, Schrøderc \& Nielsend, 2019). As altas taxas de mortalidade geralmente estão associadas não apenas a complicações relacionadas ao diagnóstico tardio, mas também à semelhança dos seus sinais e sintomas em relação a outras doenças da infância, o que implica em prejuízos na chance de cura e suspeita diagnóstica em fases mais avançadas da doença (Radhi et al., 2015; Massoud et al., 2016; Ries \& Costenaro, 2017; Herbert et al., 2018; Paiva et al., 2018). Diante disso, o reconhecimento precoce da doença pela família e, principalmente, pela equipe médica por meio da identificação dos sinais e sintomas iniciais torna-se fundamental para o tratamento oportuno e, consequentemente, melhores desfechos psicossociais e clínicos (Herbert et al., 2018).

Devido a etiologia do CIJ ser multifatorial, há uma demora no diagnóstico e consequentemente um aumento tanto da morbidade quanto da mortalidade. Sendo que sua identificação precoce melhora o prognóstico. Estudos focados especificamente no atraso do diagnóstico no CIJ são escassos, principalmente em países em desenvolvimento (Carberry et al., 2018). Portanto, objetivou-se caracterizar sinais e sintomas do CIJ através da percepção dos pais dos acometidos, assim como associar os sinais e sintomas com as variáveis sociodemográficas e clínicas no município de Aracaju, Estado de Sergipe, entre os anos 1991 e 2019.

\section{Metodologia}

Trata-se de um estudo transversal, de caráter exploratório e abordagem quantitativa, realizado no período de dezembro de 2019 em banco de dados cadastrais, padronizado pela AVOSOS, que incluem os genitores de crianças e adolescentes, entre 0 a 19 anos, em tratamento para neoplasias malignas, assistidos pela instituição, no momento da admissão.

Foram incluídas todas as crianças e os adolescentes, entre 0 a 19 anos, cadastrados no banco de dados de fevereiro de 1991 a setembro de 2019, sendo excluídos os portadores de neoplasias malignas com dados cadastrais incompletos (sem sinais e sintomas, sem classificação tumoral).

A instituição disponibilizou aos pesquisadores o banco de dados em uma planilha, omitindo a identificação nominal dos indivíduos envolvidos. As variáveis estudadas foram: idade, procedência, sinais e sintomas iniciais, tempo decorrido do início dos sintomas, para procurar o serviço de saúde e de diagnóstico, classificação da neoplasia, tratamento realizado e óbito.

Os dados foram analisados e interpretados utilizando a estatística descritiva com a caracterização exploratória das variáveis utilizando toda a amostra e dividindo-a por cada categoria da CICI. Foram testadas as diferenças entre as proporções entre indivíduos com ou sem sintomas por meio do teste $\mathrm{Z}$ para proporções. O nível de significância adotado foi de $5 \%$ e o software utilizado foi o R Core Team 2019.

O trabalho aprovado pelo Comitê de Ética e Pesquisa da Universidade Tiradentes, sob o parecer consubstanciado ${ }^{\circ}$ 3.239.059.

\section{Resultados}

O banco de dados estudado contém 763 crianças e adolescentes cadastrados em tratamento de neoplasias malignas, no período de fevereiro de 1991 a setembro de 2019. Foram selecionados 719 casos, excluindo-se 44 casos que não continham todos os dados necessários ao estudo.

Os sinais e sintomas iniciais mais frequentemente relatados pelos genitores foram a febre $(18,4 \%)$ seguida da dor $(17,5 \%)$. Em ordem decrescente foram citadas ainda especificamente a cefaleia, vômitos, astenia e a presença de tumorações. Também é comum o aparecimento de mais de um sinal e sintoma (Tabela 1). 
Tabela 1: Frequência dos sinais e sintomas relatados dos casos de neoplasias malignas infanto-juvenis cadastradas na AVOSOS, em Aracaju, Sergipe de 1991 a 2019.

\begin{tabular}{lccc}
\hline Sinais e Sintomas & $\mathbf{N}$ & \% das respostas & \% de casos \\
Febre & 285 & 18,4 & 40,0 \\
Dor & 271 & 17,5 & 38,1 \\
Cefaleia & 111 & 7,2 & 15,6 \\
Vômito & 97 & 6,3 & 13,6 \\
Astenia & 92 & 5,9 & 12,9 \\
Tumoração & 83 & 5,3 & 11,7 \\
Palidez & 72 & 4,6 & 10,1 \\
Distensão Abdominal & 66 & 4,3 & 9,3 \\
Hematoma & 63 & 4,1 & 8,8 \\
Edema & 62 & 4,0 & 8,7 \\
Tontura & 46 & 3,0 & 6,5 \\
Perda de Peso & 37 & 2,4 & 5,2 \\
Anorexia & 36 & 2,3 & 5,1 \\
Adenopatia & 35 & 2,3 & 4,9 \\
Incoordenação motora & 17 & 1,1 & 2,4 \\
Outros & 179 & 13,8 & 26,8 \\
Total & 1552 & 100,0 & 218,0 \\
\hline
\end{tabular}

Legenda: $\mathrm{n}$ - frequência absoluta. \% - frequência relativa percentual. Fonte: Banco de dados da AVOSOS (1991 a 2019).

As neoplasias malignas que mais atingiram o segmento infantojuvenil, no período analisado foram, respectivamente: Leucemias (37,6\%), Linfomas (16,8\%), Tumores do SNC (12,5\%), Tumores renais (7,2\%) e Tumores ósseos (6,8\%). As maiores frequências de óbitos dos acometidos por CIJ ocorreram nos neuroblastomas (52,2\%), tumores ósseos (51\%), tumores do SNC (46,7\%), Tumores de tecidos moles e outros sarcomas (46,2\%) e nas leucemias (39,3\%) (Tabela 2). 
Tabela 2: Frequências das neoplasias malignas infanto-juvenis e de óbitos cadastrados na AVOSOS de acordo com a CICI, em Aracaju, Sergipe de 1991 a 2019.

\begin{tabular}{lcc}
\hline CICI & $\mathbf{n}$ & $\mathbf{\%}$ \\
\hline I (Leucemias) & 270 & 37,6 \\
II (Linfomas) & 121 & 16,8 \\
III (Tumores do SNC) & 90 & 12,5 \\
IV (Neuroblastomas) & 23 & 3,2 \\
V (Retinoblastomas) & 15 & 2,1 \\
VI (Tumores Renais) & 52 & 7,2 \\
VII (Tumores Hepáticos) & 8 & 1,1 \\
VIII (Tumores ósseos) & 49 & 6,8 \\
IX (Tecidos moles e outros sarcomas) & 31 & 4,3 \\
X (Tumores de células germinativas) & 12 & 1,7 \\
XI (Outras neoplasias malignas epiteliais) & 15 & 2,1 \\
XII (Outras neoplasias malignas não especificadas) & 33 & 4,6 \\
Total & 719 & 100,0 \\
\hline Óbitos & $\mathbf{n}$ & $\mathbf{\%}$ \\
\hline I (Leucemias) & 106 & 39,3 \\
II (Linfomas) & 32 & 26,4 \\
III (Tumores do SNC) & 42 & 46,7 \\
IV (Neuroblastomas) & 12 & 52,2 \\
V (Retinoblastomas) & 4 & 26,7 \\
VI (Tumores Renais) & 11 & 21,2 \\
VII (Tumores Hepáticos) & 4 & 50,0 \\
VIII (Tumores ósseos) & 25 & 51,0 \\
IX (Tecidos moles e outros sarcomas) & 14 & 46,2 \\
X (Tumores de células germinativas) & 2 & 16,7 \\
XI (Outras neoplasias malignas epiteliais) & 3 & 20,0 \\
XII (Outras neoplasias malignas não especificadas) & 6 & 18,2 \\
Total & 261 & 36,3 \\
\hline
\end{tabular}

Legenda: (n) frequência absoluta; (\%) porcentagem.

Fonte: Banco de dados da AVOSOS (1991 a 2019).

A faixa etária dos pacientes analisados foi de 1 mês a 19 anos. O grupo que obteve a menor média de idade de acometimento correspondeu ao V (Retinoblastomas) com 3,52 anos e a maior média ficou estabelecida no grupo VIII (Tumores ósseos) com 12,8 anos (Tabela 3). 
Tabela 3 - Classificação das neoplasias malignas infantojuvenis por idade cadastradas na AVOSOS, em Aracaju, Sergipe de 1991 a 2019.

\begin{tabular}{|c|c|c|c|c|c|c|}
\hline CICI & $\mathbf{n}$ & $\begin{array}{l}\text { Média } \\
\text { (anos) }\end{array}$ & Dp & Min & Max & $\begin{array}{c}\text { Mediana } \\
\text { (anos) }\end{array}$ \\
\hline I (Leucemias) & 270 & 7,63 & 5,25 & 1 mês & 19 anos & 6 \\
\hline II (Linfomas) & 121 & 9,52 & 5,18 & 1 ano & 19 anos & 9 \\
\hline III (Tumores do SNC) & 90 & 7,42 & 4,52 & 3 meses & 19 anos & 7 \\
\hline IV (Neuroblastomas) & 23 & 3,78 & 2,95 & 3 meses & 11 anos & 3 \\
\hline V (Retinoblastomas) & 15 & 3,52 & 3,15 & 5 meses & 11 anos & 3 \\
\hline VI (Tumores Renais) & 52 & 4,02 & 3,83 & 1 mês & 16 anos & 3 \\
\hline VII (Tumores Hepáticos) & 8 & 4,46 & 2,84 & 8 meses & 9 anos & 4 \\
\hline VIII (Tumores ósseos) & 49 & 12,80 & 4,32 & 3 meses & 19 anos & 13 \\
\hline IX (Tecidos moles/sarcomas) & 31 & 9,73 & 6,13 & 9 meses & 19 anos & 10 \\
\hline $\mathbf{X}$ (Tumor células germinativas) & 12 & 5,50 & 4,64 & 1 ano & 14 anos & 4,5 \\
\hline XI (Outras neo malig epiteliais) & 15 & 9,80 & 4,21 & 4 anos & 17 anos & 10 \\
\hline $\begin{array}{l}\text { XII (Outras neo malig não } \\
\text { especificadas) }\end{array}$ & 33 & 7,87 & 5,20 & 8 meses & 19 anos & 6 \\
\hline Total & 719 & 7,88 & 5,31 & 1 mês & 19 anos & 7 \\
\hline
\end{tabular}

Legenda: $\mathrm{n}$ - frequência absoluta. \% - frequência relativa percentual; dp - desvio padrão; Min - mínimo; Max - máximo. Fonte: banco de dados da AVOSOS (1991 a 2019).

Pode-se observar que $563(79,6 \%)$ dos pais dos acometidos por CIJ procuraram o serviço de saúde em até um mês da ocorrência dos sintomas. A maioria dos diagnósticos ocorreu entre 1 a 3 meses do início dos sintomas (39,9\%), com apenas 35,4\% dos diagnósticos realizados em até um mês da sintomatologia apresentada (Tabela 4).

Tabela 4: Intervalo de tempo em procurar a Unidade Básica de Saúde e para o diagnóstico na maioria dos casos de neoplasias malignas infanto-juvenis cadastradas na AVOSOS, em Aracaju, Sergipe de 1991 a 2019.

\begin{tabular}{lcc}
\hline Intervalo de tempo em procurar a UBS & $\mathbf{n}$ & $\mathbf{\%}$ \\
Até 1 mês & 563 & 79,6 \\
1 a 3 meses & 89 & 12,6 \\
3 a 6 meses & 28 & 4,0 \\
6 a 9 meses & 8 & 1,1 \\
9 a 12 meses & 13 & 1,8 \\
> 12 meses & 6 & 0,8 \\
\hline Intervalo de tempo para o diagnóstico & 248 & 35,4 \\
Até 1 mês & 279 & 39,9 \\
1 a 3 meses & 92 & 13,1 \\
3 a 6 meses & 23 & 3,3 \\
6 a 9 meses & 23 & 3,3 \\
9 a 12 meses & 35 & 5,0 \\
\hline 12 meses & & \\
\hline
\end{tabular}

Legenda: $\mathrm{n}$ - frequência absoluta. \% - frequência relativa percentual.

Fonte: banco de dados da AVOSOS (1991 a 2019).

Os tratamentos mais realizados pelos portadores de CIJ foram, respectivamente, a quimioterapia (63,2\%) e os procedimentos cirúrgicos $(29,7 \%)$. Porém, muitos pacientes também são submetidos a terapias combinadas, sendo a quimioterapia associada à cirurgia a mais relatada. (Tabela 5). 
Tabela 5: Frequência dos tratamentos realizados pelos portadores dos casos de neoplasias malignas infanto-juvenis cadastradas na AVOSOS, em Aracaju, Sergipe de 1991 a 2019.

\begin{tabular}{lccc}
\hline Tratamento & n & \% das respostas & \% de casos \\
Quimioterapia & 594 & 63,2 & 88,5 \\
Cirurgia & 279 & 29,7 & 41,6 \\
Radioterapia & 52 & 5,5 & 7,7 \\
Outros & 15 & 1,6 & 2,2 \\
Total & 940 & 100,0 & 140,1 \\
\hline
\end{tabular}

Legenda: $\mathrm{n}$ - frequência absoluta. \% - frequência relativa percentual. Fonte: banco de dados da AVOSOS (1991 a 2019).

Ademais, foi verificada a associação de sinais e sintomas com os óbitos, faixa etária, CICI, tempo de procura pela UBS, tempo de diagnóstico e terapias utilizadas. Entre 1 a 4 anos, a febre foi o sintoma mais predominante, enquanto entre 10 e 14 anos a cefaleia predominou. Em relação ao CICI, o tipo I manifestou-se principalmente por febre, dor, astenia e palidez. No tipo II e IX, a tumoração apresentou-se como sinal mais predominante. No tipo III, a cefaleia e o vômito predominaram. Em relação ao tempo de procura pela UBS, a febre foi o sinal que levou o cuidador a procurar atendimento médico em menor tempo. Avaliando-se o tempo de diagnóstico, a febre, astenia e palidez foram os sintomas mais prevalentes em pacientes cm tempo de diagnóstico de até 3 meses. Enquanto a tumoração foi o sinal que levou a um tempo maior para o diagnóstico. A quimioterapia foi mais utilizada em crianças que apresentava febre, dor, astenia e tumoração. A cefaleia foi o sintoma que predominou nos pacientes que se submeteram a cirurgia (Tabela 6). 
Tabela 6: Associação entre febre, dor, cefaleia, vômito, astenia, tumoração e palidez e variáveis sóciodemográficas e clínicas das neoplasias malignas infanto-juvenis cadastradas na AVOSOS, em Aracaju, Sergipe de 1991 a 2019.

\begin{tabular}{|c|c|c|c|c|c|c|c|}
\hline & Febre & Dor & Cefaleia & $\begin{array}{c}\text { Vômit } \\
\text { o }\end{array}$ & Astenia & Tumoração & Palidez \\
\hline & $\mathrm{n}(\%)$ & $\mathrm{n}(\%)$ & $\mathrm{n}(\%)$ & $\mathrm{n}(\%)$ & $\mathrm{n}(\%)$ & $\mathrm{n}(\%)$ & $\mathrm{n}(\%)$ \\
\hline Óbito & $101(34,9)$ & $107(37,9)$ & $50(41)$ & $18(46,2)$ & $42(44,7)$ & $21(25,6)$ & $26(36,1)$ \\
\hline \multicolumn{8}{|l|}{ Faixa Etária } \\
\hline$<1$ ano & $12(4,2)$ & $5(1,8)$ & $1(0,9)$ & $1(2,6)$ & $7(7,5)$ & $2(2,5)$ & $5(7,1)$ \\
\hline 1-4 anos & $111(38,5)$ & $78(28,6)$ & $18(16,2)$ & $10(26,3)$ & $15(16,1)$ & $20(25,3)$ & $24(34,3)$ \\
\hline 5-9 anos & $75(26,0)$ & $73(26,0)$ & $31(27,9)$ & $11(28,9)$ & $29(31,2)$ & $23(29,1)$ & $18(25,7)$ \\
\hline 10-14 anos & $58(20,1)$ & $71(26,0)$ & $35(31,5)$ & $9(23,7)$ & $24(25,8)$ & $16(20,3)$ & $18(25,7)$ \\
\hline 15-19 anos & $32(11,1)$ & $46(16,8)$ & $26(23,4)$ & $7(18,4)$ & $18(19,4)$ & $18(22,8)$ & $5(7,1)$ \\
\hline \multicolumn{8}{|l|}{ CICI } \\
\hline I & $164(56,7)$ & $125(44,3)$ & $37(30,3)$ & $15(38,5)$ & $57(60,6)$ & $7(8,5)$ & $60(83,3)$ \\
\hline II & $41(14,2)$ & $40(14,2)$ & $8(6,6)$ & $2(5,1)$ & $11(11,7)$ & $40(48,8)$ & $6(8,3)$ \\
\hline III & $16(5,5)$ & $13(4,6)$ & $56(45,9)$ & $13(33,3)$ & $7(7,4)$ & $2(2,4)$ & $0(0)$ \\
\hline IV & $9(3,1)$ & $7(2,5)$ & $4(3,3)$ & $1(2,6)$ & $2(2,1)$ & $4(4,9)$ & $0(0)$ \\
\hline V & $3(1)$ & $3(1,1)$ & $3(2,5)$ & $0(0)$ & $1(1,1)$ & $0(0)$ & $0(0)$ \\
\hline VI & $23(8)$ & $23(8,2)$ & $0(0)$ & $2(5,1)$ & $6(6,4)$ & $4(4,9)$ & $1(1,4)$ \\
\hline VII & $2(0,7)$ & $4(1,4)$ & $2(1,6)$ & $0(0)$ & $1(1,1)$ & $1(1,2)$ & $1(1,4)$ \\
\hline VIII & $6(2,1)$ & $35(12,4)$ & $3(2,5)$ & $0(0)$ & $5(5,3)$ & $9(11)$ & $3(4,2)$ \\
\hline IX & $5(1,7)$ & $9(3,2)$ & $4(3,3)$ & $2(5,1)$ & $2(2,1)$ & $8(9,8)$ & $0(0)$ \\
\hline $\mathrm{X}$ & $3(1)$ & $5(1,8)$ & $0(0)$ & $0(0)$ & $1(1,1)$ & $2(2,4)$ & $0(0)$ \\
\hline XI & $2(0,7)$ & $1(0,4)$ & $4(3,3)$ & $0(0)$ & $0(0)$ & $2(2,4)$ & $1(1,4)$ \\
\hline XII & $15(5,2)$ & $17(6)$ & $1(0,8)$ & $4(10,3)$ & $1(1,1)$ & $3(3,7)$ & $0(0)$ \\
\hline \multicolumn{8}{|l|}{$\begin{array}{l}\text { Tempo de } \\
\text { procura UBS }\end{array}$} \\
\hline Até 3 meses & $254(88,8)$ & $228(82,3)$ & $99(81,1)$ & $33(84,6)$ & $77(82,8)$ & $58(73,4)$ & $63(88,7)$ \\
\hline 1 a 3 meses & $19(6,6)$ & $30(10,8)$ & $15(12,3)$ & $5(12,8)$ & $9(9,7)$ & $9(11,4)$ & $4(5,6)$ \\
\hline 3 a 6 meses & $8(2,8)$ & $14(5,1)$ & $5(4,1)$ & $1(2,6)$ & $3(3,2)$ & $6(7,6)$ & $2(2,8)$ \\
\hline 6 a 9 meses & $0(0)$ & $1(0,4)$ & $2(1,6)$ & $0(0)$ & $2(2,2)$ & $2(2,5)$ & $2(2,8)$ \\
\hline 9 a 12 meses & $0(0)$ & $0(0)$ & $0(0)$ & $0(0)$ & $0(0)$ & $0(0)$ & $0(0)$ \\
\hline$>12$ meses & $5(1,7)$ & $4(1,4)$ & $1(0,8)$ & $0(0)$ & $2(2,2)$ & $4(5,1)$ & $0(0)$ \\
\hline \multicolumn{8}{|l|}{ Tempo de } \\
\hline \multicolumn{8}{|l|}{ Diagnóstico } \\
\hline Até 3 meses & $128(45,4)$ & $100(36,4)$ & $43(35,5)$ & $14(36,8)$ & $48(52,2)$ & $22(28,6)$ & $35(50)$ \\
\hline 1 a 3 meses & $104(36,9)$ & $110(40)$ & $38(31,4)$ & $14(36,8)$ & $31(33,7)$ & $20(26)$ & $25(35,7)$ \\
\hline 3 a 6 meses & $31(11)$ & $37(13,5)$ & $21(17,4)$ & $6(15,8)$ & $4(4,3)$ & $15(19,5)$ & $4(5,7)$ \\
\hline 6 a 9 meses & $6(2,1)$ & $9(3,3)$ & $8(6,6)$ & $1(2,6)$ & $3(3,3)$ & $3(3,9)$ & $4(5,7)$ \\
\hline 9 a 12 meses & $0(0)$ & $2(0,7)$ & $0(0)$ & $0(0)$ & $0(0)$ & $1(1,3)$ & $0(0)$ \\
\hline$>12$ meses & $13(4,6)$ & $17(6,2)$ & $11(9,1)$ & $3(7,9)$ & $6(6,5)$ & $16(20,8)$ & $2(2,9)$ \\
\hline \multicolumn{8}{|l|}{ Terapias } \\
\hline Quimioterapia & $267(92,4)$ & $248(87,9)$ & $80(65,6)$ & $30(76,9)$ & $86(91,5)$ & $68(82,9)$ & $69(95,8)$ \\
\hline Radioterapia & $20(6,9)$ & $12(4,3)$ & $10(8,2)$ & $2(5,1)$ & $6(6,4)$ & $9(11)$ & $2(2,8)$ \\
\hline Cirurgia & $20(6,9)$ & $26(9,2)$ & $35(28,7)$ & $7(17,9)$ & $4(4,3)$ & $13(15,9)$ & $3(4,2)$ \\
\hline Outras Terapias & $3(1)$ & $5(1,8)$ & $1(0,8)$ & $0(0)$ & $1(1,1)$ & $2(2,4)$ & $0(0)$ \\
\hline Nenhuma Terapia & $9(3,1)$ & $11(3,9)$ & $11(9)$ & $2(5,1)$ & $4(4,3)$ & $2(2,4)$ & $1(1,4)$ \\
\hline
\end{tabular}

Legenda: $\mathrm{n}$ - frequência absoluta. \% - frequência relativa percentual. Em negrito, a presença do sintoma está relacionada a uma maior prevalência da classe na coluna significativamente a $5 \%$ no teste $\mathrm{Z}$ para proporções.

Fonte: banco de dados da AVOSOS (1991 a 2019).

A distensão abdominal predominou na faixa etária inferior a 4 anos e nos grupos CICI IV e VI; hematoma foi mais verificado entre 1 a 4 anos, na CICI e em pacientes submetidos a quimioterapia; edema foi mais frequente no grupo CICI IX e em neoplasias diagnosticadas mais tardiamente. Demais associações entre sinais e variáveis sociodemográficas podem ser verificadas na Tabela 7. 
Tabela 7: Associação entre distensão abdominal, hematoma, edema, tontura, perda de peso, anorexia, adenopatia e outros sintomas e variáveis sócio-demográficas e clínicas das neoplasias malignas infanto-juvenis cadastradas na AVOSOS, em Aracaju, Sergipe de 1991 a 2019.

\begin{tabular}{|c|c|c|c|c|c|c|c|c|}
\hline & $\begin{array}{c}\text { Distensã } \\
\text { o } \\
\text { Abdomi } \\
\text { nal }\end{array}$ & $\begin{array}{c}\text { Hemato } \\
\text { ma }\end{array}$ & $\begin{array}{l}\text { Edem } \\
\mathrm{a}\end{array}$ & Tontura & $\begin{array}{c}\text { Perda } \\
\text { de } \\
\text { Peso }\end{array}$ & $\begin{array}{c}\text { Anorexi } \\
\mathrm{a}\end{array}$ & $\begin{array}{c}\text { Adeno } \\
\text { patia }\end{array}$ & $\begin{array}{c}\text { Outros } \\
\text { Sintoma } \\
\text { s }\end{array}$ \\
\hline & $\mathrm{n}(\%)$ & $\mathrm{n}(\%)$ & $\mathrm{n}(\%)$ & $\mathrm{n}(\%)$ & $\mathrm{n}(\%)$ & $\mathrm{n}(\%)$ & $\mathrm{n}(\%)$ & $\mathrm{n}(\%)$ \\
\hline Óbito & $19(28,4)$ & $20(33,3)$ & $24(35,8)$ & $18(46,2)$ & $17(44,7)$ & $13(35,1)$ & $9(25)$ & $79(42,5)$ \\
\hline \multicolumn{9}{|l|}{ Faixa Etária } \\
\hline$<1$ ano & $6(9,1)$ & $0(0,0)$ & $0(0,0)$ & $0(0,0)$ & $0(0,0)$ & $0(0,0)$ & $1(3,0)$ & $10(5,7)$ \\
\hline 1-4 anos & $39(59,1)$ & $29(48,3)$ & $20(31,7)$ & $5(14,3)$ & $11(29,7)$ & $19(51,4)$ & $5(15,2)$ & $62(35,2)$ \\
\hline 5-9 anos & $14(21,2)$ & $13(21,7)$ & $14(22,2)$ & $10(28,6)$ & $12(32,4)$ & $9(24,3)$ & $11(33,3)$ & $42(23,9)$ \\
\hline 10-14 anos & $7(10,6)$ & $11(18,3)$ & $14(22,2)$ & $12(34,3)$ & $9(24,3)$ & $7(18,9)$ & $9(27,3)$ & $38(21,6)$ \\
\hline 15-19 anos & $0(0,0)$ & $7(11,7)$ & $15(23,8)$ & $8(22,9)$ & $5(13,5)$ & $2(5,4)$ & $7(21,2)$ & $24(13,6)$ \\
\hline \multicolumn{9}{|l|}{ CICI } \\
\hline I & $26(38,8)$ & $52(86,7)$ & $20(29,9)$ & $25(64,1)$ & $12(31,6)$ & $25(67,6)$ & $13(36,1)$ & $61(32,8)$ \\
\hline II & $7(10,4)$ & $4(6,7)$ & $15(22,4)$ & $3(7,7)$ & $9(23,7)$ & $2(5,4)$ & $17(47,2)$ & $20(10,8)$ \\
\hline III & $0(0)$ & $0(0)$ & $1(1,5)$ & $8(20,5)$ & $2(5,3)$ & $3(8,1)$ & $0(0)$ & $48(25,8)$ \\
\hline IV & $5(7,5)$ & $1(1,7)$ & $1(1,5)$ & $1(2,6)$ & $2(5,3)$ & $3(8,1)$ & $0(0)$ & $6(3,2)$ \\
\hline V & $0(0)$ & $0(0)$ & $0(0)$ & $1(2,6)$ & $0(0)$ & $0(0)$ & $0(0)$ & $10(5,4)$ \\
\hline VI & $19(28,4)$ & $0(0)$ & $1(1,5)$ & $0(0)$ & $5(13,2)$ & $1(2,7)$ & $0(0)$ & $11(5,9)$ \\
\hline VII & $1(1,5)$ & $0(0)$ & $0(0)$ & $0(0)$ & $1(2,6)$ & $1(2,7)$ & $0(0)$ & $0(0)$ \\
\hline VIII & $2(3)$ & $0(0)$ & $15(22,4)$ & $0(0)$ & $4(10,5)$ & $0(0)$ & $0(0)$ & $8(4,3)$ \\
\hline IX & $1(1,5)$ & $1(1,7)$ & $8(11,9)$ & $1(2,6)$ & $1(2,6)$ & $1(2,7)$ & $1(2,8)$ & $5(2,7)$ \\
\hline$X$ & $1(1,5)$ & $0(0)$ & $0(0)$ & $0(0)$ & $2(5,3)$ & $0(0)$ & $1(2,8)$ & $5(2,7)$ \\
\hline XI & $2(3)$ & $2(3,3)$ & $1(1,5)$ & $0(0)$ & $0(0)$ & $0(0)$ & $1(2,8)$ & $6(3,2)$ \\
\hline XII & $3(4,5)$ & $0(0)$ & $5(7,5)$ & $0(0)$ & $0(0)$ & $1(2,7)$ & $3(8,3)$ & $6(3,2)$ \\
\hline \multicolumn{9}{|l|}{$\begin{array}{l}\text { Tempo de } \\
\text { procura UBS }\end{array}$} \\
\hline Até 3 meses & $55(82,1)$ & $55(91,7)$ & $56(83,6)$ & $35(89,7)$ & $26(68,4)$ & $33(89,2)$ & $28(80)$ & $149(80,5)$ \\
\hline 1 a 3 meses & $8(11,9)$ & $2(3,3)$ & $8(11,9)$ & $2(5,1)$ & $4(10,5)$ & $1(2,7)$ & $3(8,6)$ & $17(9,2)$ \\
\hline 3 a 6 meses & $1(1,5)$ & $1(1,7)$ & $3(4,5)$ & $1(2,6)$ & $4(10,5)$ & $1(2,7)$ & $3(8,6)$ & $9(4,9)$ \\
\hline 6 a 9 meses & $0(0)$ & $1(1,7)$ & $0(0)$ & $1(2,6)$ & $2(5,3)$ & $1(2,7)$ & $0(0)$ & $2(1,1)$ \\
\hline 9 a 12 meses & $0(0)$ & $0(0)$ & $0(0)$ & $0(0)$ & $0(0)$ & $0(0)$ & $0(0)$ & $1(0,5)$ \\
\hline$>12$ meses & $3(4,5)$ & $1(1,7)$ & $0(0)$ & $0(0)$ & $2(5,3)$ & $1(2,7)$ & $1(2,9)$ & $7(3,8)$ \\
\hline \multicolumn{9}{|l|}{ Tempo de } \\
\hline \multicolumn{9}{|l|}{ Diagnóstico } \\
\hline Até 3 meses & $27(41,5)$ & $29(48,3)$ & $14(20,9)$ & $14(35,9)$ & $11(31,4)$ & $16(43,2)$ & $10(28,6)$ & $52(28,1)$ \\
\hline 1 a 3 meses & $25(38,5)$ & $19(31,7)$ & $29(43,3)$ & $18(46,2)$ & $15(42,9)$ & $16(43,2)$ & $14(40)$ & $83(44,9)$ \\
\hline 3 a 6 meses & $7(10,8)$ & $7(11,7)$ & $14(20,9)$ & $3(7,7)$ & $5(14,3)$ & $3(8,1)$ & $6(17,1)$ & $28(15,1)$ \\
\hline 6 a 9 meses & $3(4,6)$ & $2(3,3)$ & $2(3)$ & $3(7,7)$ & $3(8,6)$ & $2(5,4)$ & $1(2,9)$ & $7(3,8)$ \\
\hline 9 a 12 meses & $0(0)$ & $0(0)$ & $2(3)$ & $0(0)$ & $0(0)$ & $0(0)$ & $0(0)$ & $0(0)$ \\
\hline$>12$ meses & $3(4,6)$ & $3(5)$ & $6(9)$ & $1(2,6)$ & $1(2,9)$ & $0(0)$ & $4(11,4)$ & $15(8,1)$ \\
\hline \multicolumn{9}{|l|}{ Terapias } \\
\hline Quimioterapia & $61(91)$ & $55(91,7)$ & $56(83,6)$ & $33(84,6)$ & $35(92,1)$ & $34(91,9)$ & $31(86,1)$ & $146(78,5)$ \\
\hline Radioterapia & $3(4,5)$ & $0(0)$ & $2(3)$ & $3(7,7)$ & $4(10,5)$ & $2(5,4)$ & $3(8,3)$ & $15(8,1)$ \\
\hline Cirurgia & $5(7,5)$ & $0(0)$ & $4(6)$ & $4(10,3)$ & $2(5,3)$ & $4(10,8)$ & $2(5,6)$ & $33(17,7)$ \\
\hline Outras Terapias & $2(3)$ & $0(0)$ & $0(0)$ & $0(0)$ & $1(2,6)$ & $1(2,7)$ & $5(13,9)$ & $4(2,2)$ \\
\hline \multicolumn{9}{|l|}{ Nenhuma } \\
\hline Terapia & $3(4,5)$ & $5(8,3)$ & $7(10,4)$ & $3(7,7)$ & $2(5,3)$ & $1(2,7)$ & $2(5,6)$ & $13(7)$ \\
\hline
\end{tabular}

Legenda: $\mathrm{n}$ - frequência absoluta. \% - frequência relativa percentual. Em negrito, a presença do sintoma está relacionada a uma maior prevalência da classe na coluna significativamente a $5 \%$ no teste $\mathrm{Z}$ para proporções.

Fonte: banco de dados da AVOSOS (1991 a 2019). 


\section{Discussão}

No presente estudo, os sinais e sintomas mais frequentemente relatados pelos cuidadores de crianças com câncer foram a febre (18,4\%) e a dor (17,5\%). Este corrabora com o estudo de Tutelman et al. (2018), que descreveram que a dor é um dos sintomas mais comuns e angustiantes desde o diagnóstico até o fim da vida em portadores de câncer infantojuvenil. Porém, por serem sinais e sintomas inespecíficos são relatados frequentemente em doenças benignas frequentes na infância.

O CIJ mais incidente neste estudo foram as leucemias, concordando com demais estudos como o de Carvalho et al (2020), Santos Júnior et al. (2018) e Araújo et al (2020) que demonstrou as leucemias como as neoplasias mais prevalentes na pediatria, com prevalência de $18,25 \%, 36 \%$ e $43,4 \%$, respectivamente. A leucemia tem a febre e a dor como manifestações predominantes (Silva Delfin et al., 2018). Ao mesmo tempo febre é um sintoma que preocupa muito os cuidadores, levando-os a procurar atendimento médico mais rápido.

A dor foi mais frequente nos tumores hepáticos, tumores renais, tumores ósseos, tumores de células germinativas, outras neoplasias malignas epiteliais e outras neoplasias malignas não especificadas.

A cefaleia foi o registro mais encontrado nas tumorações do SNC e outras neoplasias malignas epiteliais, entrando em consenso com o estudo de Delfin BS et al (2018) no qual relata que o sintoma mais frequente do SNC, relacionado principalmente á hipertensão intracraniana é a cefaleia.

Como esperado, a tumoração foi o sinal mais prevalente nos linfomas, nos tumores de tecidos moles e sarcoma e em outras neoplasias malignas epiteliais. Estudo feito em Recife-PE relata as linfonodomegalias/tumorações como segunda manifestação, perdendo apenas para a dor (Oliveira et al, 2021).

O estudo de Mutti et al (2018), associa a demora do diagnóstico com o aumento da morbimortalidade, visto que, geralmente, quanto mais avançada está a doença, menores são as chances de cura e maiores são as sequelas decorrentes do tratamento que acaba sendo mais agressivo. Entretanto, neste estudo, na maioria das vezes, o CIJ levou de 1 a 3 meses para ser diagnosticado e se comparado com pesquisas como o de Silva et al (2011) que o tempo entre início dos sintomas e o diagnóstico para a maioria das crianças, foi mais de 60 dias, o diagnóstico neste estudo é considerado precoce, ajudando no melhor prognóstico do paciente.

No presente trabalho, as frequências de óbitos por CIJ foram, respectivamente, neuroblastomas, tumores ósseos, tumores do SNC, tumores de tecidos moles e outros sarcomas e leucemias. Discordando dos dados apresentados por Carvalho et al (2020), em que as neoplasias infanto-juvenis com maior mortalidade foram as leucemias, os linfomas, os tumores do SNC e os neuroblastomas e dos dados de Spironello et al. (2020), em que as neoplasias do SNC (36,27\%) foram as que mais levaram a óbito, seguidas dos neuroblastomas $(32,13 \%)$, das leucemias $(29,31 \%)$ e das neoplasias de tecidos moles $(21,56 \%)$. Apesar dos tipos de câncer serem concordantes, as frequências mostraram divergência. Em função dos sintomas que levaram a procura de serviços médicos com maior precocidade coincidirem com os sintomas frequentemente relatados nas leucemias, esse tipo de câncer foi diagnosticado em período inicial, permitindo mais sobrevida para a população estudada.

No que tange à idade, a idade média de incidência foi de 7,63 anos $( \pm 5,25)$. Estando com uma média menor de faixa etária em relação ao estudo realizado em Petrolina (PE) em que a idade média foi de 11,6 anos (Silva et al 2018). Não há muitos estudos atuais relacionando a idade média do acometimento do CIJ, tendo uma divergência em relação aos estudos já existentes. A menor média de idade de acometimento correspondeu ao retinoblastomas (3,52 anos) e a maior média ficou estabelecida nos tumores ósseos (12,8 anos), concordando com a literatura que considera estes tumores uma neoplasia tipicamente de adolescente (INCA, 2016; Santos Júnior et al., 2018; Carvalho et al., 2020).

Quanto ao intervalo de tempo para o diagnóstico, merece atenção o diagnóstico, em até 30 dias do primeiro sintoma em 35\% dos casos registrados, percentual de diagnóstico precoce duas vezes maior encontrado pelo INCA (2016), que teve 16,1\% dos casos com diagnóstico dentro de 30 dias da primeira consulta médica. Esse reconhecimento contribui positivamente 
com a redução das complicações e da mortalidade, melhorando o prognóstico e a qualidade de vida na oncologia pediátrica. No estudo de Sá et al. (2019), oito participantes obtiveram o diagnóstico do câncer em menos de oito dias, entretanto, dois casos chamaram a atenção, dentre os quais, um que recebeu o diagnóstico com dez meses e o outro com um ano após os primeiros sinais e sintomas.

Com relação ao tratamento, no presente estudo, o mais utilizado foi a quimioterapia sendo empregada de maneira isolada ou associada a outros tratamentos. Corroborando com outros trabalhos (Carvalho et al., 2020; Santos Júnior CJ et al., 2018; Benchaya, Ferreira \& Brasiliense, 2014; INCA, 2016; Mills et al., 2018; Mutti et al.,2018; Bauer et al., 2015; Oliveira et al., 2019) que também apontaram a quimioterapia como o tratamento antineoplásico mais utilizado no CIJ. Também semelhante a pesquisa de Mutti et al. (2018), apontaram a radioterapia como a terapêutica menos utilizada entre os pacientes oncológicos infantis, sendo associada a outros tratamentos como a quimioterapia e /ou cirurgia.

A família pode ser a primeira a suspeitar dos sinais e dos sintomas do CIJ e contribuir positivamente no diagnóstico precoce. Isso porque, quando os pais percebem algo errado, buscam atendimento médico nas unidades básicas de saúde, nos serviços de pronto-atendimento, nas consultas agendadas em convênios médicos e particulares (Friestino \& Moreira Filho, 2016). É preocupante ter encontrado que $20 \%$ das crianças com câncer foram levadas a um serviço de saúde após um mês do primeiro sintoma. As crianças estão, geralmente, sob cuidados de seus pais e familiares e as principais manifestações percebidas pelos pais desta investigação (febre e dor) são inespecíficas, e talvez, consideradas de menor gravidade, o que pode ter retardado a procura pelo atendimento médico. Podemos considerar a dificuldade no acesso também um fator associado a este atraso. Infelizmente, o presente estudo não buscou as causas para tal fato.

\section{Conclusão}

Conclui-se que os principais sinais e sintomas percebidos pelos pais foram a febre e a dor, seguidos pela cefaleia, vômitos, astenia e presença de tumorações.

O maior percentual de procura pelos serviços de saúde pelos pais dos acometidos se estabeleceu em até um mês da ocorrência dos sintomas, porém a maioria do estabelecimento dos diagnósticos ocorreu entre 1 a 3 meses após o início da sintomatologia.

O reconhecimento de sinais e sintomas pelos pais dos acometimentos com câncer infanto-juvenil que traz a importância deste trabalho, visto que, qualquer aparecimento e/ou mudança no estado geral da criança levam os genitores a procuraram o serviço médico. Assim, se trabalharmos para que os pais e familiares consigam identificar esses sintomas (mesmo que inespecíficos) a procuraram algum serviço de saúde o mais rápido possível, iremos contribuir para o melhor prognóstico e diminuição da morbimortalidade do câncer pediátrico.

\section{Referências}

Araújo, M. A. S., Jurema, G. L., da Silva, A. D., \& Gonçalves, E. (2020). Câncer infantil: perfil epidemiológico em população atendida por hospital de referência no Piauí. Revista Eletrônica Acervo Saúde/Electronic Journal Collection Health| ISSN, $2178,2091$.

Bauer, D. F. V., Ferrari, R. A. P., dos Reis, T. B., \& Tacla, M. T. G. M. (2015). Crianças com câncer: caracterização das internações em um hospital escola público. Semina: Ciências Biológicas e da Saúde, 36(1Supl), 9-16.

Bravo, L. E., García, L. S., Collazos, P., Aristizabal, P., \& Ramirez, O. (2013). Descriptive epidemiology of childhood cancer in Cali: Colombia 19772011. Colombia Médica: CM, 44(3), 155.

Carberry, A. R., Hanson, K., Flannery, A., Fischer, M., Gehlbach, J., Diamond, C., \& Wald, E. R. (2018). Diagnostic error in pediatric cancer. Clinical pediatrics, 57(1), 11-18.

Carvalho, W. M. O., da Silva, R. N., de Carvalho, M. B. F., Batista, T. F., de Carvalho Nascimento, R. I. F., Nascimento, M. V. R., \& Jeraldo, V. D. L. S. (2020). Aspectos epidemiológicos do câncer infantojuvenil em uma capital do nordeste brasileiro. Revista Eletrônica Acervo Saúde, 12 (11), e4045-e4045.

Delfin, B. S., Arca, H.L., Costa, L.B.Z., Pereira, S. G. (2018). Detecção Precoce do Câncer Infantil em Foz do Iguaçu, PR. Pleiade, 12(S1): 29-33. 
Feliciano, S. V. M., Santos, M. O., \& Pombo-de-Oliveira, M. S. (2018). Incidência e mortalidade por câncer entre crianças e adolescentes: uma revisão narrativa. Revista Brasileira de Cancerologia, 64(3), 389-396.

Friestino, J. K. O., \& de Carvalho Moreira Filho, D. (2016). Panorama do Câncer em crianças e adolescentes sob a perspectiva da Saúde Coletiva. Revista Baiana de Saúde Pública, 40(2).

Herbert, A., Lyratzopoulos, G., Whelan, J., Taylor, R. M., Barber, J., Gibson, F., \& Fern, L. A. (2018). Diagnostic timeliness in adolescents and young adults with cancer: a cross-sectional analysis of the BRIGHTLIGHT cohort. The Lancet Child \& Adolescent Health, 2(3), 180-190.

INCA. Instituto Nacional de Câncer. Câncer Infantojuvenil. Tipos de Câncer / Instituto Nacional de Câncer. Rio de Janeiro; INCA; 2019.

Lilja-Fischer, J. K., Schrøder, H., \& Nielsen, V. E. (2019). Pediatric malignancies presenting in the head and neck. International journal of pediatric otorhinolaryngology, 118, 36-41.

Massoud, M., Del Bufalo, F., Musolino, A. M. C., Schingo, P. M., Gaspari, S., Pisani, M., \& Raucci, U. (2016). Myeloid sarcoma presenting as low back pain in the pediatric emergency department. The Journal of emergency medicine, 51(3), 308-314.

Mutti, C. F., da Cruz, V. G., Santos, L. F., de Araújo, D., Cogo, S. B., \& Neves, E. T. (2018). Perfil clínico-epidemiológico de crianças e adolescentes com câncer em um serviço de oncologia. Revista Brasileira de Cancerologia, 64(3), 293-300.

Oliveira, A. T., de Sousa, M. N. A., Maia, P. C. G. G. S., Bezerra, A. L. D., de Moura, B. C. P., de Lucena Santos, E. V., \& do Egypto, I. A. S. (2019). Perfil epidemiológico do câncer infantil na Paraíba. Revista Eletrônica Acervo Saúde, 11(16), e1568-e1568.

Oliveira, J. V. L., da Silva, N. C., de França, A. M. M., dos Anjos, M. K. S., da Silva, F. C., de Abreu Silva, M. A., \& Renesto, H. M. F. (2021). Perfil de crianças e adolescentes com câncer diagnosticadas através do projeto fique atento: pode ser câncer na Cidade do Recife-PE. Research, Society and Development, 10(5), e19610514738-e19610514738.

Paiva, M. P. F. (2018). Estudo do tempo entre o diagnóstico e o início do tratamento de crianças com câncer em um centro de referência da Paraíba.

Radhi, M., Fulbright, J. M., Ginn, K. F., \& Guest, E. M. (2015). Childhood cancer for the primary care physician. Primary Care: Clinics in Office Practice, 42(1), 43-55.

Ries, P. K., \& Costenaro, R. G. S. (2017). Fatores relacionados ao diagnóstico tardio das neoplasias na infância e adolescência. Disciplinarum Scientia| Saúde, 18(1), 111-121.

Sá, A. C. S., da Silva, A. C. S. S., \& Góes, F. G. B. (2019). Diagnóstico do Câncer Infantojuvenil: O Caminho Percorrido Pelas Famílias. Revista de Pesquisa Cuidado é Fundamental Online, 11(5), 1180-1187.

Santos Júnior, C. J., Romão, C. M. D. S. B., Alves, M. J. R. G., Batinga, A. M. C. S., da Silva Gomes, V. M., Araújo, N. S., \& Leite, L. A. C. (2018). Características clínico-epidemiológicas do câncer infantojuvenil no estado de Alagoas, Brasil. Revista de Medicina, 97(5), 454-460.

Silva Delfin, B., Arca, H. L., Costa, L. B. Z., \& Pereira, S. G. (2018). Deteç̧ão Precoce do Câncer Infantil em Foz do Iguaçu, PR. Revista Pleiade, 12(24), 2933 .

Silva, M. A. V. (2011). Câncer infanto juvenil: trajetória dos casos com suspeição realizada em unidades não especializadas no município do Rio de Janeiro (Doctoral dissertation).

Silva, M. G. P., Bedor, C. N. G., Alencar, K. M. D. S. A., Curado, M. P., \& Moura, L. T. R. D. (2018). Tendências da morbimortalidade por câncer infantojuvenil em um polo de fruticultura irrigada. Cadernos Saúde Coletiva, 26, 38-44.

Spironello, R. A., Silva-Comar, F. M. S., Cardia, G. F. E., Janeiro, V., Pedroso, R. B., \& Cuman, R. K. N. (2020). Mortalidade infantil por câncer no Brasil. Saúde e Pesquisa, 13(1), 115-122.

Tutelman, P. R., Chambers, C. T., Stinson, J. N., Parker, J. A., Fernandez, C. V., Witteman, H. O., \& Irwin, K. (2018). Pain in children with cancer. The Clinical journal of pain, 34(3), 198-206. 